\title{
Is nutritional labeling associated with individual health? The effects of labeling- based awareness on dyslipidemia risk in a South Korean population
}

Jong Yeob Kim¹, Ki Hong Kweon ${ }^{1}$, Min Jae Kim', Eun-Cheol Park ${ }^{2,3}$, Suk-Yong Jang ${ }^{2,3}$, Woorim Kim ${ }^{3,4}$ and Kyu-Tae Han ${ }^{3,4^{*}}$

\begin{abstract}
Background: In 1995, the South Korean government made nutrition labeling compulsory, which has positively impacted patients with certain chronic diseases, such as dyslipidemia. We investigated the association between nutrition labeling-based awareness and the risk of dyslipidemia among individuals not yet diagnosed.

Methods: Our study used data from the fifth Korea National Health and Nutrition Examination Surveys administered during 2010-2014 ( $n=17,687$ ). We performed multiple or logistic regression analysis to examine the association between nutritional analysis and various outcome variables.

Results: Approximately $70 \%$ of the respondents $(n=11,513)$ were familiar with nutrition labeling, of which $20 \%(n=3172)$ decided what food to buy based on that information. This awareness yielded mostly positive results on outcome indicators, such as triglyceride and high-density lipoprotein cholesterol levels. In general, individuals who used nutritional labels to make decisions regarding food purchases had a lower risk of dyslipidemia than individuals who did not (OR: 0.806, $95 \%$ Cl: 0.709-0.917).

Conclusion: Utilizing nutrition labels for making food choices correlated with a lower risk of dyslipidemia in certain subgroups. Based on our findings, we recommend that health policymakers and medical professionals consider promoting nutrition labeling as an alternative method for managing certain chronic diseases in South Korean patients.
\end{abstract}

Keywords: Nutrition labeling, Health policy perception, Dyslipidemia, Hyperlipidemia

\section{Background}

During the past 30 years, South Korea has experienced evolving health care perspectives, with a recent focus on chronic diseases. Although many health care professionals have studied treatment options extensively, some chronic diseases persist in South Korean patients [1]. Therefore, developing prevention strategies for managing risk factors, such as hypertension, diabetes

\footnotetext{
* Correspondence: kthan@yuhs.ac

${ }^{3}$ Institute of Health Services Research, Yonsei University, Seoul, Republic of Korea

${ }^{4}$ Department of Public Health, Graduate School, Yonsei University, Seoul, Republic of Korea

Full list of author information is available at the end of the article
}

mellitus, and dyslipidemia, may be important for controlling these diseases [2-4].

Dyslipidemia is a state of abnormal amounts of lipids in the blood and is characterized by conditions such as hypercholesterolemia, hypertriglyceridemia, increased low-density lipoprotein (LDL) cholesterolemia, and decreased high-density lipoprotein (HDL) cholesterolemia [5]. Dyslipidemia can be managed by diet, exercise, and sometimes drug injections, depending on the health of the patient [6]. However, based on previous studies in South Korea, the prevalence rate of dyslipidemia has gradually increased since 2000 [7]. Although not necessarily harmful itself, the condition is a major risk factor for various cardiovascular 
diseases (CVD) [8]. Mortality due to CVD has also increased in recent years, making it the second most common cause of death in South Korea [9]. Therefore, it is essential to investigate alternatives for effectively preventing and/or managing dyslipidemia.

In 1995, the South Korean government made nutrition labeling compulsory. Nutrition labeling is a type of food labeling [10] that describes the nutritional properties of processed foods to help consumers make a reasonable choice in purchasing food based on its nutritional values [11]. Labeling also protects consumers from dishonest advertisement by providing exact nutrition information. Previous studies show that nutrition labeling affects food intake with respect to total fat, carbohydrates, and saturated fat and that awareness of nutrition facts and may be helpful in managing certain chronic diseases [12-14].

Because nutrition labeling has since expanded in South Korea, some positive effects on patients with chronic diseases, particularly dyslipidemia, have been linked closely to dietary patterns $[15,16]$. Despite increased dyslipidemia prevalence and the expansion of nutrition labeling in South Korea, few studies have investigated their relationship. As introducing the nutrition labelling system in South Korea, we expected that the health information related to food consumption would be well provided to South Korean. Therefore, South Korean would easily access to health information which might be helpful in well managing their health compared to past. Based on our hypothesis that nutrition labeling may help prevent dyslipidemia, we analyzed the potential association between nutrition labeling-based awareness and the prevalence of dyslipidemia among individuals not yet diagnosed.

\section{Methods}

\section{Study population}

This study used data from the fifth Korea National Health and Nutrition Examination Surveys (KNHANES V/VI 2010-14), which are cross-sectional questionnaires that have been administered annually since 1998 by the Korea Centers for Disease Control and Prevention (KCDC) to assess the health and nutritional status of the Korean population. This survey is composed of three parts: Health Interview Survey, Health Examination, and Nutrition Survey. The health examination survey collected the information about anthropometric index, blood pressure, blood test, urine test, dental examination, pulmonary function test, optical test, and hearing test. These tests were performed through visiting examination using vehicle for health examination. The nutrition survey was conducted through additional visiting research of investigator after Health Interview Survey and Health Examination. The nutrition survey including average amount of daily fat intake was consisted to dietary pattern, dietary supplements, nutrition knowledge, food safety, food intake of the day before survey ( $24 \mathrm{~h}$ recall method), and food frequency questionnaire. A stratified multi-stage cluster-sampling design was used to obtain a nationally representative sample from the three parts of the survey. The overall response rates were $81.9 \%$ in 2010, $80.4 \%$ in 2011, $80.0 \%$ in 2012, $79.3 \%$ in 2013, and $77.8 \%$ in 2014 and included 41,101 total respondents. Individuals not tested for dyslipidemia indicators and those under the age of 30 were excluded from the study. In addition, we excluded respondents diagnosed with dyslipidemia before the survey. Thus, we included 17,687 eligible participants in the study.

\section{Variables analyzed}

The outcome variables analyzed in this study included four indicators of dyslipidemia: total cholesterol (TC), LDL cholesterol, HDL cholesterol, and triglyceride (TG) levels. Although TC, TG, and HDL cholesterol levels were measured on the day of investigation. This blood test was measured through fasting blood test (minimum $8 \mathrm{~h}$ and recommended $12 \mathrm{~h}$ after eating). The LDL cholesterol levels were not measured, so were instead calculated using the Friedewald formula. This methods also relatively efficient methods than the ultracentrifugal measurement of LDL cholesterol [17]. We first considered each indicator as a continuous variable and then defined dyslipidemia as the presence of at least one indicator meeting the following diagnostic criteria: $\mathrm{TC} \geq 200 \mathrm{mg} / \mathrm{dL}$, LDL cholesterol $\geq 130 \mathrm{mg} / \mathrm{dL}$, HDL cholesterol $\leq 40 \mathrm{mg} / \mathrm{dL}$, or $\mathrm{TG} \geq 150 \mathrm{mg} / \mathrm{dL}$ [18].

The primary independent variable was the respondents' awareness regarding nutrition labeling, which we defined as one of three levels: 1) "unaware of nutrition facts (lowest awareness)"; 2) "aware of nutrition facts but does not check them when making food purchase/checks nutrition facts but does not make labeling-dependent purchase decisions"; or 3) "checks nutrition facts and makes labelingdependent purchase decisions (highest awareness)".

We included other independent variables to investigate the association between labeling awareness and dyslipidemia. These additional variables were sex, age, educational level, economic activity, household income, body mass index (BMI), aerobic exercise habits, smoking status, high risk drinking, family history of hyperlipidemia, stress awareness, subjective health, average amount of daily fat intake, frequency of eating out, and survey year [19-21]. Age was divided by 10year increments or grouped as more than 60 years old. Educational level was classified as no high school graduation, bachelor's degree, and master's degree or above. BMI was categorized into three groups based on obesity criteria in South Korea $(<23,23-25$, 
Table 1 General characteristics of study population by awareness regarding nutrition labelling in in this study

\begin{tabular}{|c|c|c|c|c|c|c|c|}
\hline \multirow{2}{*}{$\begin{array}{l}\text { Awareness regarding nutrition } \\
\text { labelling } \\
\text { Variables }\end{array}$} & \multicolumn{2}{|c|}{$\begin{array}{l}\text { Checks nutrition facts and makes } \\
\text { labeling-dependent purchase } \\
\text { decisions }\end{array}$} & \multicolumn{2}{|c|}{$\begin{array}{l}\text { Checks nutrition facts but does not make } \\
\text { labeling-dependent purchase decisions/ } \\
\text { Aware of nutrition facts but does not check } \\
\text { them when making food purchase decisions }\end{array}$} & \multicolumn{2}{|c|}{$\begin{array}{l}\text { Unaware of } \\
\text { nutrition facts }\end{array}$} & \multirow[t]{2}{*}{$P$-value } \\
\hline & N/Mean & $\% / S D$ & N/Mean & $\% / S D$ & N/Mean & $\% / S D$ & \\
\hline \multicolumn{8}{|l|}{ Sex } \\
\hline Male & 645 & 8.76 & 3,739 & 50.78 & 2,979 & 40.46 & $<.0001$ \\
\hline Female & 2,527 & 24.48 & 4,602 & 44.58 & 3,195 & 30.95 & \\
\hline \multicolumn{8}{|l|}{ Age (years) } \\
\hline $30-39$ & 1,406 & 34.19 & 2,402 & 58.41 & 304 & 7.39 & $<.0001$ \\
\hline $40-49$ & 1,014 & 25.76 & 2,367 & 60.14 & 555 & 14.10 & \\
\hline $50-59$ & 524 & 13.88 & 2,001 & 53.02 & 1,249 & 33.09 & \\
\hline $60+$ & 228 & 3.89 & 1,571 & 26.79 & 4,066 & 69.33 & \\
\hline \multicolumn{8}{|l|}{ Educational level } \\
\hline Under high school graduation & 1,313 & 11.25 & 4,881 & 41.83 & 5,476 & 46.92 & $<.0001$ \\
\hline Bachelor's degree & 1,635 & 30.91 & 3,035 & 57.37 & 620 & 11.72 & \\
\hline Master's degree or above & 224 & 30.81 & 425 & 58.46 & 78 & 10.73 & \\
\hline \multicolumn{8}{|l|}{ Economic activity } \\
\hline Unemployed & 1,389 & 20.23 & 2,706 & 39.41 & 2,772 & 40.37 & $<.0001$ \\
\hline Employed & 1,783 & 16.48 & 5,635 & 52.08 & 3,402 & 31.44 & \\
\hline \multicolumn{8}{|l|}{ Household income } \\
\hline Low & 172 & 5.09 & 869 & 25.70 & 2,340 & 69.21 & $<.0001$ \\
\hline Mid-low & 715 & 15.92 & 2,124 & 47.29 & 1,652 & 36.78 & \\
\hline Mid-high & 1,085 & 22.04 & 2,599 & 52.79 & 1,239 & 25.17 & \\
\hline High & 1,200 & 24.53 & 2,749 & 56.19 & 943 & 19.28 & \\
\hline \multicolumn{8}{|l|}{ BMI } \\
\hline$<23$ & 1,629 & 20.62 & 3,814 & 48.28 & 2,456 & 31.09 & $<.0001$ \\
\hline $23-25$ & 687 & 16.21 & 1,951 & 46.04 & 1,600 & 37.75 & \\
\hline$>25$ & 856 & 15.42 & 2,576 & 46.41 & 2,118 & 38.16 & \\
\hline \multicolumn{8}{|l|}{ Aerobic exercise habits } \\
\hline Yes & 937 & 21.97 & 2,115 & 49.59 & 1,213 & 28.44 & $<.0001$ \\
\hline No & 2,235 & 16.65 & 6,226 & 46.39 & 4,961 & 36.96 & \\
\hline \multicolumn{8}{|l|}{ Smoking status } \\
\hline Non-smoker & 2,821 & 19.73 & 6,536 & 45.71 & 4,943 & 34.57 & $<.0001$ \\
\hline Smoker & 351 & 10.36 & 1,805 & 53.29 & 1,231 & 36.34 & \\
\hline \multicolumn{8}{|l|}{ High risk drinking } \\
\hline No & 2,935 & 18.39 & 7,375 & 46.22 & 5,646 & 35.38 & $<.0001$ \\
\hline Yes & 237 & 13.69 & 966 & 55.81 & 528 & 30.50 & \\
\hline \multicolumn{8}{|l|}{ Family history for hyperlipidemia } \\
\hline No & 2,915 & 17.22 & 7,941 & 46.91 & 6,073 & 35.87 & $<.0001$ \\
\hline Yes & 257 & 33.91 & 400 & 52.77 & 101 & 13.32 & \\
\hline \multicolumn{8}{|l|}{ Survey year } \\
\hline 2010 & 725 & 18.28 & 1,721 & 43.39 & 1,520 & 38.33 & $<.0001$ \\
\hline 2011 & 623 & 15.73 & 1,735 & 43.81 & 1,602 & 40.45 & \\
\hline 2012 & 621 & 17.25 & 1,675 & 46.54 & 1,303 & 36.20 & \\
\hline
\end{tabular}


Table 1 General characteristics of study population by awareness regarding nutrition labelling in in this study (Continued)

\begin{tabular}{|c|c|c|c|c|c|c|c|}
\hline 2013 & 590 & 18.64 & 1,671 & 52.78 & 905 & 28.58 & \\
\hline 2014 & 613 & 20.46 & 1,539 & 51.37 & 844 & 28.17 & \\
\hline \multicolumn{8}{|l|}{ Stress awareness } \\
\hline Low & 2,348 & 17.35 & 6,369 & 47.05 & 4,820 & 35.61 & \multirow[t]{2}{*}{$<.0001$} \\
\hline High & 824 & 19.86 & 1,972 & 47.52 & 1,354 & 32.63 & \\
\hline \multicolumn{8}{|l|}{ Subjective health status } \\
\hline Good & 1,187 & 20.41 & 2,941 & 50.56 & 1,689 & 29.04 & \multirow[t]{3}{*}{$<.0001$} \\
\hline Normal & 1,611 & 18.44 & 4,239 & 48.51 & 2,888 & 33.05 & \\
\hline Bad & 374 & 11.94 & 1,161 & 37.07 & 1,597 & 50.99 & \\
\hline Average amount of daily fat intake & 46.39 & 0.77 & 46.34 & 0.51 & 33.16 & 0.55 & $<.0001$ \\
\hline \multicolumn{8}{|l|}{ The frequency of eating out } \\
\hline Less than four times a week & 2,182 & 18.01 & 4,972 & 41.04 & 4,961 & 40.95 & \multirow[t]{3}{*}{$<.0001$} \\
\hline More than five times a week & 990 & 17.77 & 3,369 & 60.46 & 1,213 & 21.77 & \\
\hline Total & 3,172 & 17.93 & 8,341 & 47.16 & 6,174 & 34.91 & \\
\hline
\end{tabular}

and $>25)$. Aerobic exercise habits were based on the amount of aerobic exercise per week, with $150 \mathrm{~min}$ of exercise as the cutoff. The smoking status was defined as follows. Smoker group included the current smoker regardless the amount of smoking. Non-smoker group included the ex-smoker and people who have never smoke in their life. The high risk drinking was defined as people who consume more than seven (for males) or five (for females) drinks on a single occasion at least twice a week. The average amount of daily fat intake was calculated based on food intake of the day before survey ( $24 \mathrm{~h}$ recall method). Respondents were recorded the information about food intake of the day before survey, and investigator calculated the nutrient component based on this information. The frequency of eating out was categorized based on five times a week. Stress awareness was defined as the respondents' daily stress awareness and was classified as "high" or "low". Subjective health status was classified as "bad," "normal," or "good."

\section{Statistical analysis}

We first examined the distribution of values by frequency and percentage for categorical variables or mean and standard deviation for continuous variables, showed the association between other independent variables and awareness of nutrition labelling. Next, we performed ANOVA for continuous variables to determine their relationship with the independent variables by comparing the means and standard deviations of the outcome variables. We also performed Chi-square tests to determine relationships with dyslipidemia diagnosis. Finally, multiple regression analysis was used to examine the association between awareness of nutrition labeling and dyslipidemia indicators while controlling for potential confounding (independent) variables described above. We then performed logistic regression analysis of dyslipidemia risk based on the four dyslipidemia indicators. In addition, we carried out subgroup multiple logistic regression analysis by sex, age, educational level, BMI, and subjective health status to examine differences in nutrition labeling-mediated awareness and dyslipidemia risk. Sampling weights assigned to each participant were applied in the analyses to generalize the sampled data.

\section{Results}

The data used in this study included 17,687 unique responses to the KNHANES V/VI from 2010 to 2014. Table 1 shows the general characteristics of our study participants by awareness of nutrition labelling. Approximately $70 \%$ of respondents were aware of nutrition labeling, but most did not actively check nutrition labels or make food purchasing decisions based on nutrition labels. Only about $20 \%$ of these respondents made nutrition label-dependent food purchasing decisions. Females were more frequently in higher awareness level in nutrition labelling than males. The people with younger age, higher educational level, and higher income were more recognized for nutrition labelling than others. In addition, people who had more healthy behaviors were more frequent in higher awareness of nutrition labelling.

Table 2 shows associations between the independent and outcome variables. The average values for dyslipidemia indicators (TC, TG, HDL cholesterol, and LDL cholesterol) were 190.88, 137.42, 50.86, and $112.54 \mathrm{mg} /$ $\mathrm{dL}$, respectively. Individuals with higher awareness of nutrition labeling had positive association with low TC, 
Table 2 The association between awareness on nutrition labelling and 4 indicators related to dyslipidemia or diagnosis of dyslipidemia

\begin{tabular}{|c|c|c|c|c|c|c|c|c|c|c|c|c|c|c|c|c|c|}
\hline \multirow[t]{3}{*}{ Variables } & \multirow{2}{*}{\multicolumn{3}{|c|}{$\begin{array}{l}\text { Total cholesterol } \\
\text { (mg/dL) }\end{array}$}} & \multirow{2}{*}{\multicolumn{3}{|c|}{$\begin{array}{l}\text { Triglyceride } \\
(\mathrm{mg} / \mathrm{dL})\end{array}$}} & \multirow{2}{*}{\multicolumn{3}{|c|}{$\begin{array}{l}\text { HDL cholesterol } \\
(\mathrm{mg} / \mathrm{dL})\end{array}$}} & \multirow{2}{*}{\multicolumn{3}{|c|}{$\begin{array}{l}\text { LDL cholesterol } \\
(\mathrm{mg} / \mathrm{dL})\end{array}$}} & \multicolumn{4}{|c|}{ Dyslipidemia } & \multirow[t]{3}{*}{$P$-value } \\
\hline & & & & & & & & & & & & & \multicolumn{2}{|c|}{ Positive } & \multicolumn{2}{|c|}{ Negative } & \\
\hline & Mean & SD & $P$-value & Mean & SD & $P$-value & Mean & SD & $P$-value & Mean & SD & $P$-value & $N$ & $\%$ & $N$ & $\%$ & \\
\hline \multicolumn{18}{|l|}{ Awareness regarding nutrition labelling } \\
\hline $\begin{array}{l}\text { Checks nutrition facts and makes } \\
\text { labeling-dependent purchase decisions }\end{array}$ & 188.53 & 34.01 & 0.0399 & 111.89 & 79.41 & $<.0001$ & 55.48 & 12.75 & $<.0001$ & 110.68 & 30.10 & 0.0006 & 1,536 & 48.42 & 1,636 & 51.58 & $<.0001$ \\
\hline $\begin{array}{l}\text { Checks nutrition facts but does not } \\
\text { make labeling-dependent purchase } \\
\text { decisions/Aware of nutrition facts but } \\
\text { does not check them when making } \\
\text { food purchase decisions }\end{array}$ & 191.32 & 34.24 & & 129.82 & 102.12 & & 52.80 & 12.52 & & 112.55 & 31.57 & & 4,778 & 57.28 & 3,563 & 42.72 & \\
\hline Unaware of nutrition facts & 192.54 & 36.35 & & 144.53 & 109.29 & & 50.13 & 12.25 & & 113.50 & 33.74 & & 4,108 & 66.54 & 2,066 & 33.46 & \\
\hline \multicolumn{18}{|l|}{ Sex } \\
\hline Male & 189.06 & 34.48 & $<.0001$ & 155.43 & 123.33 & $<.0001$ & 48.90 & 11.82 & $<.0001$ & 109.08 & 33.39 & $<.0001$ & 4,777 & 64.88 & 2,586 & 35.12 & $<.0001$ \\
\hline Female & 192.80 & 35.24 & & 114.85 & 78.70 & & 54.81 & 12.58 & & 115.02 & 30.92 & & 5,645 & 54.68 & 4,679 & 45.32 & \\
\hline \multicolumn{18}{|l|}{ Age (years) } \\
\hline $30-39$ & 183.28 & 33.23 & $<.0001$ & 116.04 & 97.17 & $<.0001$ & 54.78 & 12.67 & $<.0001$ & 105.30 & 29.70 & $<.0001$ & 1,816 & 44.16 & 2,296 & 55.84 & $<.0001$ \\
\hline $40-49$ & 190.31 & 33.50 & & 130.36 & 113.12 & & 53.24 & 12.41 & & 110.99 & 31.25 & & 2,151 & 54.65 & 1,785 & 45.35 & \\
\hline $50-59$ & 199.50 & 34.47 & & 142.52 & 109.54 & & 52.56 & 12.75 & & 118.43 & 33.11 & & 2,595 & 68.76 & 1,179 & 31.24 & \\
\hline $60+$ & 192.14 & 36.17 & & 136.74 & 89.46 & & 49.90 & 12.18 & & 114.89 & 32.61 & & 3,860 & 65.81 & 2,005 & 34.19 & \\
\hline \multicolumn{18}{|l|}{ Educational level } \\
\hline Under high school graduation & 192.88 & 35.56 & 0.1972 & 136.69 & 104.97 & 0.0105 & 51.73 & 12.58 & 0.1640 & 113.82 & 33.02 & 0.0338 & 7,343 & 62.92 & 4,327 & 37.08 & $<.0001$ \\
\hline Bachelor's degree & 187.73 & 33.61 & & 121.24 & 94.43 & & 53.75 & 12.57 & & 109.74 & 30.09 & & 2,679 & 50.64 & 2,611 & 49.36 & \\
\hline Master's degree or above & 190.55 & 33.42 & & 128.79 & 93.64 & & 52.14 & 12.55 & & 112.66 & 30.07 & & 400 & 55.02 & 327 & 44.98 & \\
\hline \multicolumn{18}{|l|}{ Economic activity } \\
\hline Unemployed & 191.26 & 36.26 & 0.5049 & 125.39 & 87.09 & 0.0150 & 52.62 & 12.87 & 0.0188 & 113.57 & 32.13 & 0.1766 & 4,024 & 58.60 & 2,843 & 41.40 & 0.4833 \\
\hline Employed & 191.23 & 34.14 & & 135.77 & 109.81 & & 52.17 & 12.44 & & 111.90 & 32.08 & & 6,398 & 59.13 & 4,422 & 40.87 & \\
\hline \multicolumn{18}{|l|}{ Household income } \\
\hline Low & 191.86 & 36.26 & 0.5209 & 140.45 & 96.60 & 0.2011 & 50.15 & 12.60 & 0.0143 & 113.62 & 33.65 & 0.3923 & 2,249 & 66.52 & 1,132 & 33.48 & $<.0001$ \\
\hline Mid-low & 191.04 & 35.62 & & 133.31 & 110.04 & & 52.20 & 12.58 & & 112.18 & 32.03 & & 2,634 & 58.65 & 1,857 & 41.35 & \\
\hline Mid-high & 190.33 & 34.21 & & 128.97 & 101.42 & & 52.99 & 12.49 & & 111.55 & 32.33 & & 2,750 & 55.86 & 2,173 & 44.14 & \\
\hline High & 191.92 & 34.22 & & 127.06 & 97.02 & & 53.36 & 12.59 & & 113.15 & 30.80 & & 2,789 & 57.01 & 2,103 & 42.99 & \\
\hline \multicolumn{18}{|l|}{ BMl } \\
\hline$<23$ & 185.60 & 33.75 & $<.0001$ & 106.94 & 79.58 & $<.0001$ & 55.72 & 13.07 & $<.0001$ & 108.50 & 30.34 & $<.0001$ & 3,666 & 46.41 & 4,233 & 53.59 & $<.0001$ \\
\hline
\end{tabular}


Table 2 The association between awareness on nutrition labelling and 4 indicators related to dyslipidemia or diagnosis of dyslipidemia (Continued)

\begin{tabular}{|c|c|c|c|c|c|c|c|c|c|c|c|c|c|c|c|c|c|}
\hline $23-25$ & 192.73 & 34.30 & & 136.59 & 100.29 & & 51.06 & 11.92 & & 114.35 & 31.64 & & 2,683 & 63.31 & 1,555 & 36.69 & \\
\hline$>25$ & 198.14 & 35.85 & & 163.34 & 119.96 & & 48.53 & 11.11 & & 116.93 & 34.14 & & 4,073 & 73.39 & 1,477 & 26.61 & \\
\hline \multicolumn{18}{|l|}{ Aerobic exercise habits } \\
\hline Yes & 190.61 & 33.52 & 0.4412 & 126.55 & 96.47 & $<.0001$ & 53.42 & 12.90 & $<.0001$ & 111.87 & 31.27 & 0.9488 & 2,425 & 56.86 & 1,840 & 43.14 & 0.0016 \\
\hline No & 191.45 & 35.43 & & 133.39 & 103.28 & & 52.01 & 12.50 & & 112.76 & 32.36 & & 7,997 & 59.58 & 5,425 & 40.42 & \\
\hline \multicolumn{18}{|l|}{ Smoking status } \\
\hline Non-smoker & 191.20 & 34.87 & $<.0001$ & 123.16 & 89.01 & $<.0001$ & 52.98 & 12.51 & $<.0001$ & 113.59 & 31.11 & 0.2760 & 8,152 & 57.01 & 6,148 & 42.99 & $<.0001$ \\
\hline Smoker & 191.41 & 35.42 & & 167.97 & 137.71 & & 49.67 & 12.67 & & 108.15 & 35.69 & & 2,270 & 67.02 & 1,117 & 32.98 & \\
\hline \multicolumn{18}{|l|}{ High risk drinking } \\
\hline No & 190.96 & 34.90 & $<.0001$ & 125.77 & 89.46 & $<.0001$ & 52.13 & 12.44 & $<.0001$ & 113.68 & 31.34 & $<.0001$ & 9,254 & 58.00 & 6,702 & 42.00 & $<.0001$ \\
\hline Yes & 193.88 & 35.57 & & 186.79 & 169.14 & & 54.38 & 13.89 & & 102.14 & 36.89 & & 1,168 & 67.48 & 563 & 32.52 & \\
\hline \multicolumn{18}{|l|}{ Family history for hyperlipidemia } \\
\hline No & 191.12 & 34.91 & $<.0001$ & 132.05 & 102.08 & 0.1179 & 52.24 & 12.58 & 0.1999 & 112.46 & 32.11 & 0.0020 & 9,995 & 59.04 & 6,934 & 40.96 & 0.1381 \\
\hline Yes & 194.05 & 36.41 & & 124.93 & 92.97 & & 54.65 & 13.00 & & 114.41 & 32.06 & & 427 & 56.33 & 331 & 43.67 & \\
\hline \multicolumn{18}{|l|}{ Survey year } \\
\hline 2010 & 190.41 & 35.86 & 0.0245 & 130.30 & 98.76 & 0.0539 & 52.71 & 12.77 & $<.0001$ & 111.64 & 32.60 & 0.0032 & 2,294 & 57.84 & 1,672 & 42.16 & 0.0189 \\
\hline 2011 & 192.84 & 36.05 & & 132.72 & 106.89 & & 52.93 & 12.80 & & 113.36 & 32.66 & & 2,352 & 59.39 & 1,608 & 40.61 & \\
\hline 2012 & 191.79 & 34.76 & & 130.01 & 98.86 & & 51.46 & 12.45 & & 114.33 & 31.93 & & 2,187 & 60.77 & 1,412 & 39.23 & \\
\hline 2013 & 190.80 & 34.06 & & 133.48 & 106.04 & & 52.15 & 12.30 & & 111.95 & 32.09 & & 1,879 & 59.35 & 1,287 & 40.65 & \\
\hline 2014 & 190.05 & 33.47 & & 132.59 & 97.21 & & 52.36 & 12.62 & & 111.17 & 30.81 & & 1,710 & 57.08 & 1,286 & 42.92 & \\
\hline \multicolumn{18}{|l|}{ Stress awareness } \\
\hline Low & 191.18 & 34.87 & 0.1373 & 131.38 & 99.14 & 0.3396 & 52.23 & 12.58 & 0.9746 & 112.67 & 32.04 & 0.2953 & 8,020 & 59.25 & 5,517 & 40.75 & 0.1178 \\
\hline High & 191.46 & 35.33 & & 132.92 & 109.72 & & 52.73 & 12.69 & & 112.14 & 32.32 & & 2,402 & 57.88 & 1,748 & 42.12 & \\
\hline \multicolumn{18}{|l|}{ Subjective health status } \\
\hline Good & 191.50 & 34.39 & 0.0008 & 126.37 & 95.89 & 0.0031 & 53.29 & 12.74 & $<.0001$ & 112.93 & 31.31 & 0.0005 & 3,316 & 57.01 & 2,501 & 42.99 & $<.0001$ \\
\hline Normal & 191.20 & 34.43 & & 133.28 & 106.44 & & 52.25 & 12.57 & & 112.30 & 32.02 & & 5,173 & 59.20 & 3,565 & 40.80 & \\
\hline Bad & 190.88 & 37.50 & & 137.42 & 98.28 & & 50.86 & 12.33 & & 112.54 & 33.77 & & 1,933 & 61.72 & 1,199 & 38.28 & \\
\hline \multicolumn{18}{|l|}{ The frequency of eating out } \\
\hline Less than four times a week & 191.43 & 35.53 & 0.0007 & 128.23 & 96.74 & 0.8791 & 52.55 & 12.76 & 0.9080 & 113.24 & 32.19 & 0.0001 & 7,138 & 58.92 & 4,977 & 41.08 & 0.9811 \\
\hline More than five times a week & 190.83 & 33.75 & & 139.38 & 111.40 & & 51.90 & 12.28 & & 111.05 & 31.86 & & 3,284 & 58.94 & 2,288 & 41.06 & \\
\hline Total & 191.243 & 34.976 & & 131.74 & 101.717 & & 52.347 & 12.611 & & 112.548 & 32.105 & & 10,422 & 58.92 & 7,265 & 41.08 & \\
\hline
\end{tabular}


Table 3 The results of multiple regression or logistic regression analysis to examine the association between awareness on nutrition labelling and outcome variables

\begin{tabular}{|c|c|c|c|c|c|c|c|c|c|c|c|c|c|c|c|c|}
\hline \multirow[t]{2}{*}{ Variables } & \multicolumn{3}{|c|}{$\begin{array}{l}\text { Total cholesterol } \\
(\mathrm{mg} / \mathrm{dL})\end{array}$} & \multicolumn{3}{|c|}{$\begin{array}{l}\text { Triglyceride } \\
\text { (mg/dL) }\end{array}$} & \multicolumn{3}{|c|}{$\begin{array}{l}\text { HDL cholesterol } \\
(\mathrm{mg} / \mathrm{dL})\end{array}$} & \multicolumn{3}{|c|}{$\begin{array}{l}\text { LDL cholesterol } \\
(\mathrm{mg} / \mathrm{dL})\end{array}$} & \multicolumn{4}{|c|}{ Dyslipidemia } \\
\hline & $\bar{\beta}$ & SE & $P$-value & $\beta$ & SE & $P$-value & $\bar{\beta}$ & SE & $P$-value & $\bar{\beta}$ & SE & $P$-value & OR & $95 \% \mathrm{C}$ & & $P$-value \\
\hline \multicolumn{17}{|l|}{ Awareness on nutrition labelling } \\
\hline $\begin{array}{l}\text { Checks nutrition facts and makes } \\
\text { labeling-dependent purchase decisions }\end{array}$ & 0.837 & 1.056 & 0.4280 & -11.803 & 3.061 & 0.0001 & 1.259 & 0.357 & 0.0004 & 1.938 & 0.994 & 0.0515 & 0.806 & 0.709 & 0.917 & 0.0011 \\
\hline $\begin{array}{l}\text { Checks nutrition facts but does not } \\
\text { make labeling-dependent purchase } \\
\text { decisions/Aware of nutrition facts but } \\
\text { does not check them when making } \\
\text { food purchase decisions }\end{array}$ & 2.350 & 0.783 & 0.0028 & -7.170 & 2.725 & 0.0086 & 0.799 & 0.249 & 0.0014 & 2.985 & 0.774 & 0.0001 & 0.919 & 0.828 & 1.020 & 0.1110 \\
\hline Unaware of nutrition facts & Ref & - & - & Ref & - & - & Ref & - & - & Ref & - & - & 1.000 & - & - & - \\
\hline \multicolumn{17}{|l|}{ Sex } \\
\hline Male & -5.197 & 0.833 & $<.0001$ & 27.026 & 2.565 & $<.0001$ & -6.089 & 0.279 & $<.0001$ & -4.513 & 0.768 & $<.0001$ & 1.395 & 1.265 & 1.537 & $<.0001$ \\
\hline Female & Ref & - & - & Ref & - & - & Ref & - & - & Ref & - & - & 1.000 & - & - & - \\
\hline \multicolumn{17}{|l|}{ Age (years) } \\
\hline $30-39$ & -10.395 & 1.105 & $<.0001$ & -6.634 & 3.267 & 0.0426 & 1.822 & 0.372 & $<.0001$ & -10.890 & 1.056 & $<.0001$ & 0.497 & 0.432 & 0.572 & $<.0001$ \\
\hline $40-49$ & -5.299 & 1.035 & $<.0001$ & 6.350 & 3.465 & 0.0672 & 0.706 & 0.367 & 0.0544 & -7.275 & 1.001 & $<.0001$ & 0.678 & 0.596 & 0.772 & $<.0001$ \\
\hline $50-59$ & 3.804 & 0.985 & 0.0001 & 11.906 & 3.065 & 0.0001 & 1.012 & 0.322 & 0.0018 & 0.411 & 0.940 & 0.6623 & 1.168 & 1.025 & 1.331 & 0.0198 \\
\hline $60+$ & Ref & - & - & Ref & - & - & Ref & - & - & Ref & - & - & 1.000 & - & - & - \\
\hline \multicolumn{17}{|l|}{ Educational level } \\
\hline Under high school graduation & -2.553 & 1.535 & 0.0966 & 1.013 & 4.692 & 0.8291 & 0.221 & 0.548 & 0.6871 & -2.976 & 1.456 & 0.0413 & 0.983 & 0.801 & 1.206 & 0.8674 \\
\hline Bachelor's degree & -2.206 & 1.508 & 0.1438 & -3.499 & 4.603 & 0.4474 & 0.206 & 0.537 & 0.7017 & -1.712 & 1.417 & 0.2273 & 0.954 & 0.776 & 1.173 & 0.6558 \\
\hline Master's degree or above & Ref & - & - & Ref & - & - & Ref & - & - & Ref & - & - & 1.000 & - & - & - \\
\hline \multicolumn{17}{|l|}{ Economic activity } \\
\hline Unemployed & 0.749 & 0.794 & 0.3461 & 4.398 & 2.187 & 0.0446 & -0.464 & 0.251 & 0.0653 & 0.333 & 0.729 & 0.6480 & 1.153 & 1.049 & 1.267 & 0.0031 \\
\hline Employed & Ref & - & - & Ref & - & - & Ref & - & - & Ref & - & - & 1.000 & - & - & - \\
\hline \multicolumn{17}{|l|}{ Household income } \\
\hline Low & 0.258 & 1.039 & 0.8042 & 2.602 & 3.436 & 0.4491 & -0.585 & 0.389 & 0.1331 & 0.323 & 1.031 & 0.7544 & 1.076 & 0.946 & 1.223 & 0.2677 \\
\hline Mid-low & -0.302 & 0.876 & 0.7302 & -0.916 & 3.057 & 0.7644 & -0.196 & 0.303 & 0.5180 & 0.077 & 0.805 & 0.9237 & 0.926 & 0.829 & 1.035 & 0.1771 \\
\hline Mid-high & 0.064 & 0.848 & 0.9399 & -2.031 & 2.702 & 0.4524 & 0.012 & 0.269 & 0.9648 & 0.458 & 0.808 & 0.5705 & 0.953 & 0.858 & 1.059 & 0.3729 \\
\hline High & Ref & - & - & Ref & - & - & Ref & - & - & Ref & - & - & 1.000 & - & - & - \\
\hline \multicolumn{17}{|l|}{ BMI } \\
\hline$<23$ & -13.918 & 0.749 & $<.0001$ & -55.011 & 2.572 & $<.0001$ & 6.944 & 0.253 & $<.0001$ & -9.860 & 0.725 & $<.0001$ & 0.306 & 0.280 & 0.335 & $<.0001$ \\
\hline $23-25$ & -7.322 & 0.869 & $<.0001$ & -27.998 & 3.190 & $<.0001$ & 2.754 & 0.265 & $<.0001$ & -4.477 & 0.795 & $<.0001$ & 0.566 & 0.509 & 0.629 & $<.0001$ \\
\hline$>25$ & Ref & - & - & Ref & - & - & Ref & - & - & Ref & - & - & 1.000 & - & - & - \\
\hline
\end{tabular}


Table 3 The results of multiple regression or logistic regression analysis to examine the association between awareness on nutrition labelling and outcome variables (Continued)

\begin{tabular}{|c|c|c|c|c|c|c|c|c|c|c|c|c|c|c|c|c|}
\hline \multicolumn{17}{|l|}{ Aerobic exercise habits } \\
\hline Yes & Ref & - & - & Ref & - & - & Ref & - & - & Ref & - & - & 1.000 & - & - & - \\
\hline No & 0.783 & 0.752 & 0.2981 & 10.527 & 2.478 & $<.0001$ & -1.456 & 0.255 & $<.0001$ & 0.134 & 0.717 & 0.8517 & 1.090 & 0.992 & 1.199 & 0.0731 \\
\hline \multicolumn{17}{|l|}{ Smoking status } \\
\hline Non-smoker & Ref & - & - & Ref & - & - & Ref & - & - & Ref & - & - & 1.000 & - & - & - \\
\hline Smoker & 3.364 & 0.916 & 0.0003 & 26.004 & 3.516 & $<.0001$ & -1.325 & 0.301 & $<.0001$ & -0.512 & 0.910 & 0.5739 & 1.445 & 1.292 & 1.616 & $<.0001$ \\
\hline \multicolumn{17}{|l|}{ High risk drinking } \\
\hline No & Ref & - & - & Ref & - & - & Ref & - & - & Ref & - & - & 1.000 & - & - & - \\
\hline Yes & 2.614 & 1.123 & 0.0202 & 41.059 & 5.895 & $<.0001$ & 4.954 & 0.366 & $<.0001$ & -10.553 & 1.190 & $<.0001$ & 1.229 & 1.066 & 1.416 & 0.0046 \\
\hline \multicolumn{17}{|l|}{ Family history for hyperlipidemia } \\
\hline No & Ref & - & - & Ref & - & - & Ref & - & - & Ref & - & - & 1.000 & - & - & - \\
\hline Yes & 6.016 & 1.501 & $<.0001$ & 5.369 & 4.264 & 0.2083 & 0.477 & 0.531 & 0.3687 & 4.465 & 1.380 & 0.0013 & 1.307 & 1.096 & 1.560 & 0.0028 \\
\hline \multicolumn{17}{|l|}{ Survey year } \\
\hline 2010 & 0.362 & 1.093 & 0.7404 & -9.251 & 3.187 & 0.0038 & 1.093 & 0.354 & 0.0021 & 1.120 & 1.007 & 0.2663 & 0.998 & 0.878 & 1.134 & 0.9711 \\
\hline 2011 & 1.526 & 1.090 & 0.1619 & -6.695 & 3.336 & 0.0451 & 1.137 & 0.349 & 0.0012 & 1.728 & 1.003 & 0.0854 & 1.000 & 0.876 & 1.142 & 0.9992 \\
\hline 2012 & 1.877 & 1.114 & 0.0922 & -5.973 & 3.523 & 0.0904 & -0.092 & 0.389 & 0.8141 & 3.163 & 1.055 & 0.0028 & 1.117 & 0.975 & 1.278 & 0.1106 \\
\hline 2013 & -0.479 & 1.062 & 0.6523 & -4.680 & 3.494 & 0.1807 & 0.513 & 0.353 & 0.1464 & -0.056 & 1.028 & 0.9567 & 1.030 & 0.900 & 1.179 & 0.6653 \\
\hline 2014 & Ref & - & - & Ref & - & - & Ref & - & - & Ref & - & - & 1.000 & - & - & - \\
\hline \multicolumn{17}{|l|}{ Stress awareness } \\
\hline Low & Ref & - & - & Ref & - & - & Ref & - & - & Ref & - & - & 1.000 & - & - & - \\
\hline High & 0.443 & 0.757 & 0.5589 & 2.219 & 2.994 & 0.4588 & 0.281 & 0.263 & 0.2850 & -0.282 & 0.732 & 0.6998 & 0.994 & 0.907 & 1.089 & 0.8906 \\
\hline \multicolumn{17}{|l|}{ Subjective health status } \\
\hline Good & 0.979 & 1.014 & 0.3347 & -8.120 & 3.109 & 0.0092 & 1.710 & 0.330 & $<.0001$ & 0.894 & 0.853 & 0.2949 & 0.942 & 0.829 & 1.070 & 0.3556 \\
\hline Normal & 0.972 & 0.978 & 0.3209 & -2.164 & 2.945 & 0.4627 & 0.761 & 0.299 & 0.0109 & 0.643 & 0.855 & 0.4521 & 1.056 & 0.937 & 1.190 & 0.3686 \\
\hline Bad & Ref & - & - & Ref & - & - & Ref & - & - & Ref & - & - & 1.000 & - & - & - \\
\hline Average amount of daily fat intake & 0.040 & 0.011 & 0.0002 & -0.030 & 0.036 & 0.4076 & 0.008 & 0.003 & 0.0139 & 0.038 & 0.010 & 0.0002 & 1.000 & 0.999 & 1.001 & 0.9479 \\
\hline \multicolumn{17}{|l|}{ The frequency of eating out } \\
\hline Less than four times a week & Ref & - & - & Ref & - & - & Ref & - & - & Ref & - & - & 1.000 & - & - & - \\
\hline More than five times a week & 1.374 & 0.801 & 0.0867 & -2.755 & 2.835 & 0.3314 & 0.074 & 0.258 & 0.7748 & 1.851 & 0.794 & 0.0199 & 1.043 & 0.943 & 1.153 & 0.4132 \\
\hline
\end{tabular}


low TG, high HDL cholesterol, low LDL cholesterol, and less diagnosis of dyslipidemia than individuals with lower awareness. Likewise, subjects with dyslipidemia were more likely to have lower awareness of nutrition labeling. In addition, older or male individuals were more frequently diagnosed with dyslipidemia, as were subjects with lower socio-economic status, educational level, or household income.

Table 3 shows results of our multiple and logistic regression analysis to investigate the association between awareness of nutrition labeling and outcome variables related to dyslipidemia. Individuals with higher awareness of nutrition labeling had lower TG and higher HDL cholesterol levels than those with lower awareness, although we observed some negative associations between awareness and TC and LDL cholesterol levels. Male or older individuals generally had association with high risk levels of four indicators, while individuals with healthy behaviors had association with low risk levels of those. The results of our logistic regression analysis to examine the association between awareness of nutrition labeling and risk of dyslipidemia show that individuals with higher awareness of nutrition labelling had a lower risk of dyslipidemia than individuals who did not. Risk of dyslipidemia was also higher in males, older participants, and individuals with unhealthy behaviors.

We also performed subgroup multiple logistic regression analysis to examine possible associations between nutrition labeling awareness and the risk of dyslipidemia with respect to sex, age, educational level, BMI, subjective health status, and the frequency of eating out. Although the interactions between subgroup variables and labeling awareness were only analyzed for sex and age, we did note positive associations between low risk of dyslipidemia and higher awareness in each group. In general, these positive association were more noticeable in males, younger individuals, those with the low educational level, obese participants, and those with the less than four times a week of eating out (Figs. 1 and 2).

\section{Discussion}

After 1995, nutrition labeling was mandated by the South Korean government to improve consumer information regarding food purchases. Its expansion since then is expected to positively impact the overall health status in South Korea, especially in patients with certain chronic diseases [10]. Thus, we hypothesized that

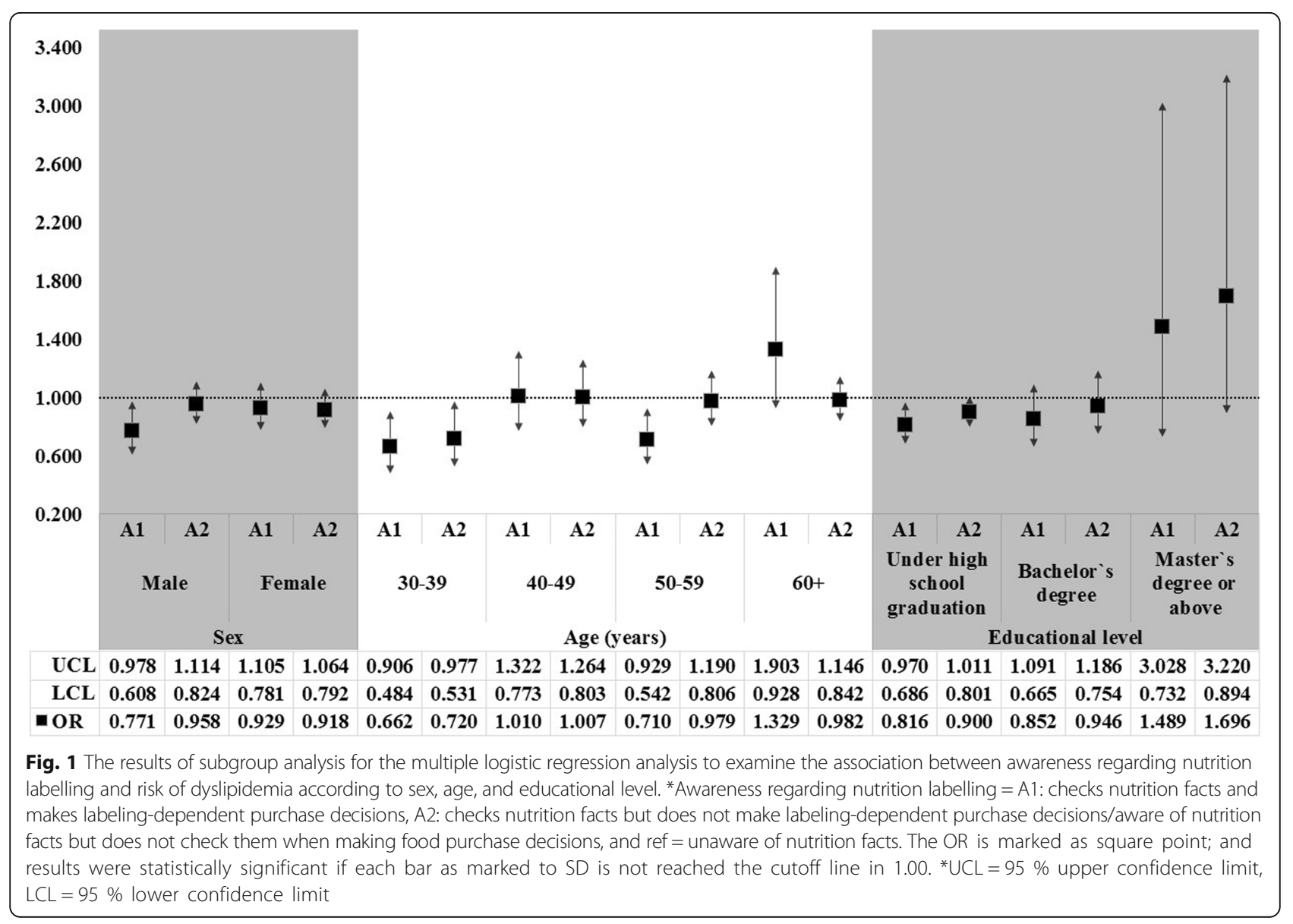




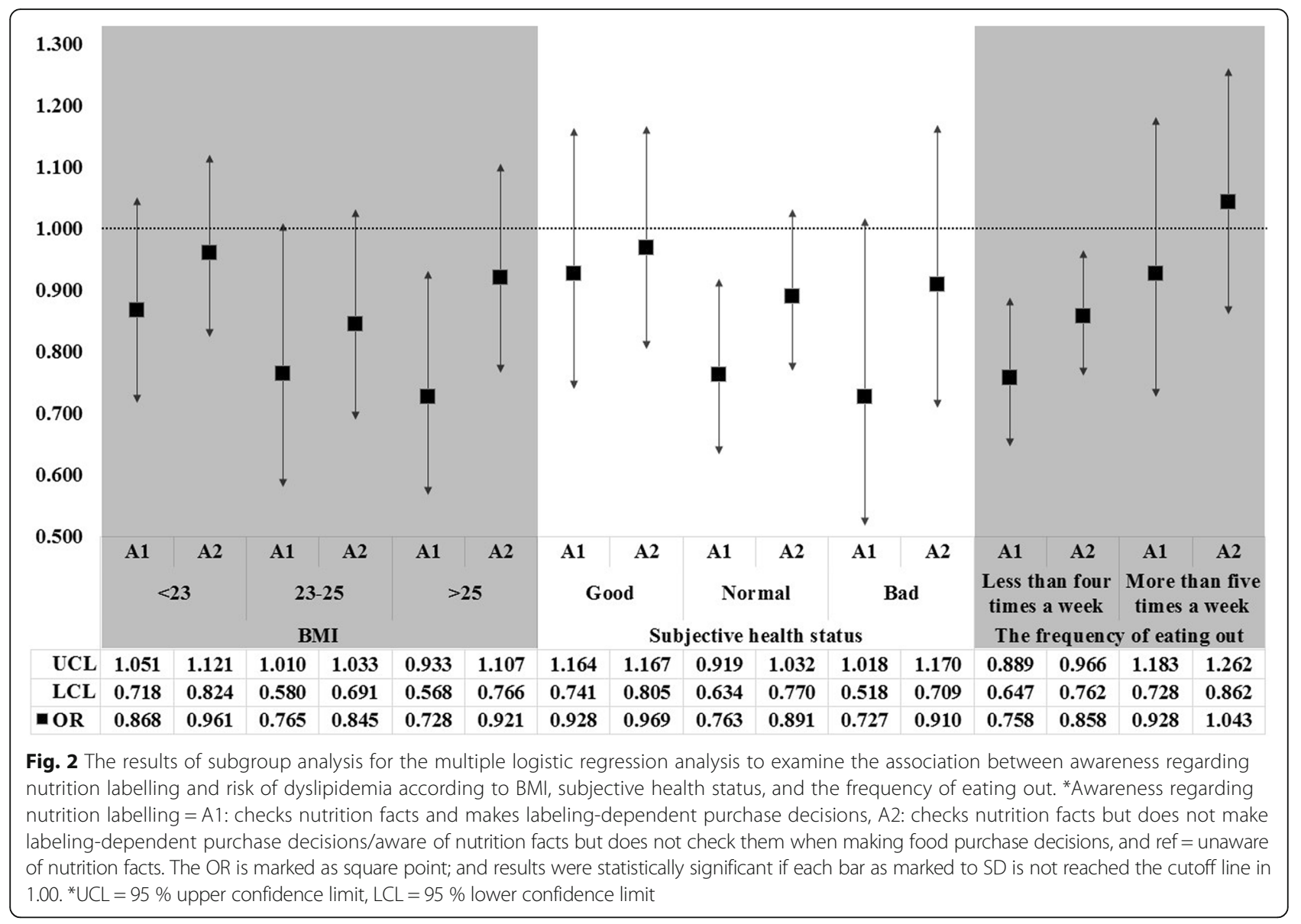

awareness of nutrition labeling significantly affects dietrelated health status, particularly dyslipidemia, and explored possible associations between awareness level and risk of dyslipidemia in individuals not yet diagnosed.

Our findings indicate that a higher awareness level was inversely related to the risk of dyslipidemia, especially with respect to TG and HDL cholesterol indicators [22]. Previous studies have already shown that nutrition labeling is positively associated with patient selfmanagement of chronic diseases, such as the changing of their dietary habits. In addition, introducing nutrition labeling may reduce obesity and promote certain healthy behaviors $[10,23]$. However, simply introduction of the labeling cannot be effective without a detailed review of how people perceive and use the system [24]. Therefore, we focused on people's self-reported awareness level of nutrition labeling rather than only examining the effects of its initial implementation. We observed similar trends to those in previous studies, but considering the poor management of dyslipidemia and mortality due to CVD in many patients, our findings could provide an effective prophylactic alternative for control of dyslipidemia.

Our subgroup analysis showed other interesting findings, such as the positive impact of higher labeling awareness in younger individuals, likely due to their general concern regarding diet choices [25]. Therefore, more public health promotion of nutrition labeling should be provided for elderly populations. Differences by sex regarding the impact of nutrition labeling were significant in only males. This also similar with reason due to age, the females had more attention for manage their health and body shape than males. In addition, there were greater impact by higher awareness of nutrition labelling than others. The nutrition labelling system in South Korea was applied into food materials for home cooking as well as meals sold by a restaurant. Based on results, the introduction of food labelling system in South Korea might be helpful in improving the health behavior of South Korean when choice the food materials for home cooking rather than eating out. Also, such results might be caused by differences of attention for health, because the people with less eating out had more attention for manage their and their family's health. Because nutrition labeling appeared to have a greater impact in individuals with lower educational level, perhaps introduction of the system has improved accessibility of health information for economically vulnerable populations [25]. The impact was also greater in 
individuals with poor health, such as those with obesity [13]. These results should motivate health professionals and policymakers to consider the positive effects of nutrition labeling awareness when establishing health policies or programs for specific populations [26]. Moreover, by promoting the advantages of nutrition labeling awareness, we expected that more remarkable improvements of health status in South Korean will be observed.

Our study had several strengths compared with previous studies. First, we used nationwide sampling data during a 5-year period, so our results are helpful in establishing long-term health policy at the national level. Second, to our knowledge, our study is the first to specifically investigate the association between awareness and utilization of nutrition labeling information and the risk of dyslipidemia in South Korean individuals. Third, our results suggest that public perception of new health policies is important for determining their long-term success rather than only shortly after their introduction [24, 27]. Finally, we considered socioeconomic status and health behaviors, such as smoking, alcohol intake, fat intake, and aerobic workout habits, to minimize the effects of confounding variables on our observed results.

However, our study also has limitations. Because the data used in this study were cross-sectional, rather than longitudinal, some concerns about causal relationships between labeling awareness and outcome variables were present. To minimize these concerns, we excluded respondents who were already diagnosed with dyslipidemia and defined dyslipidemia based on their results on the day of investigation. Second, we calculated the respondents' LDL cholesterol levels using the Friedewald formula because these data were not directly collected as part of our study [28]. The indirect measurement of LDL cholesterol may result in underestimation, so some LDL cholesterol-related results may not be accurate. Finally, the impact of labeling awareness led to some inconsistent trends with some indicators, possibly due to the method of measurement used. Therefore, further studies using data with more detailed measurements are needed.

Despite such limitations, our findings suggest that high awareness and active utilization of nutrition labeling were inversely associated with risk of dyslipidemia, especially in vulnerable populations and younger participants, as they may be more attentive to their health status than others. Based on these results, health policymakers and professionals should consider promoting nutrition labeling awareness as an alternative for managing dyslipidemia in South Korean patients.

\section{Conclusion}

The awareness of nutrition labeling had positive outcomes for TG and HDL cholesterol levels related to dyslipidemia.
In addition, the active utilization of nutrition labeling was associated with a low risk of dyslipidemia. Based on our findings, health policymakers and professionals should develop effective alternatives such as promoting the use of nutrition labeling for the management of chronic diseases in South Korea.

\begin{abstract}
Abbreviations
ANOVA: Analysis of variance; BMI: Body mass index; Cl: Confidence interval; CVD: Cardiovascular diseases; HDL: High-density lipoprotein; KCDC: Korea Centers for disease control and prevention; KNHANES: Korea National Health and Nutrition Examination Surveys; LDL: Low-density lipoprotein; OR: Odds ratio; SD: Standard deviation; SE: Standard error; TC: Total cholesterol; TG: Triglyceride
\end{abstract}

\section{Acknowledgement}

No specific funding supported this study.

\section{Availability of data and materials}

The KNHANES was openly available in https://knhanes.cdc.go.kr/knhanes/eng/ index.do after submitting e-mail address and registering short-form information.

\section{Authors' contributions}

$J Y K, K H K$, and MJK designed the study, collected data, performed statistical analyses, and wrote the manuscript. SYJ, ECP, and KTH contributed to the discussion and reviewed and edited the manuscript. KTH is the guarantor of this work and as such, had full access to all the data in the study and takes responsibility for the integrity of the data and the accuracy of the data analysis. The text in this document has been checked by at least two professional editors who are native English speakers. In addition, WK provided re-editing services for our manuscript to improve quality of scientific writing. All authors read and approved the final manuscript.

\section{Competing interests}

The authors declare that they have no competing interests.

\section{Consent for publication}

Not applicable.

\section{Ethics approval and consent to participate}

These data was approved by the KCDC Institutional Review Board, and all participants provided written informed consent (2010-02CON-21-C, 201102CON-06-C, 2012-01-EXP-01-2C, 2013-07CON-03-4C, and 2014-12EXP-03-5C).

\section{Author details}

${ }^{1}$ Premedical Courses, Yonsei University College of Medicine, Seoul, Republic of Korea. ${ }^{2}$ Department of Preventive Medicine, Yonsei University Graduate School, Seoul, Republic of Korea. Institute of Health Services Research, Yonsei University, Seoul, Republic of Korea. ${ }^{4}$ Department of Public Health, Graduate School, Yonsei University, Seoul, Republic of Korea.

Received: 25 June 2016 Accepted: 9 September 2016

Published online: 15 September 2016

\section{References}

1. Kim E-J, Yoon S-J, Jo M-W, Kim H-J. Measuring the burden of chronic diseases in Korea in 2007. Public Health. 2013;127(9):806-13.

2. Thankappan K, Shah B, Mathur P, Sarma P, Srinivas G, Mini G, Daivadanam $M$, Soman B, Vasan RS. Risk factor profile for chronic non-communicable diseases: results of a community-based study in Kerala, India. Indian J Med Res. 2010;131(1):53.

3. Egede LE. Effect of comorbid chronic diseases on prevalence and odds of depression in adults with diabetes. Psychosom Med. 2005;67(1):46-51.

4. Zimmet P, King H, Björntorp S. Obesity, hypertension, carbohydrate disorders and the risk of chronic diseases. Is there any epidemiological evidence for integrated prevention programmes? Med J Aust. 1986;145(6):256-9. 262.

5. Genest J, McPherson R, Frohlich J, Anderson T, Campbell N, Carpentier A, Couture P, Dufour R, Fodor G, Francis GA. 2009 Canadian Cardiovascular Society/Canadian guidelines for the diagnosis and treatment of dyslipidemia 
and prevention of cardiovascular disease in the adult-2009 recommendations. Can J Cardiol. 2009;25(10):567-79.

6. Varady KA, Jones PJ. Combination diet and exercise interventions for the treatment of dyslipidemia: an effective preliminary strategy to lower cholesterol levels? J Nutr. 2005;135(8):1829-35.

7. Roh E, Ko S-H, Kwon H-S, Kim NH, Kim JH, Kim CS, Song K-H, Won JC, Kim DJ, Choi SH. Prevalence and management of dyslipidemia in Korea: Korea National Health and Nutrition Examination Survey during 1998 to 2010. Diabetes Metab J. 2013;37(6):433-49.

8. Genest J, Frohlich J, Fodor G, McPherson R. Recommendations for the management of dyslipidemia and the prevention of cardiovascular disease: summary of the 2003 update. Can Med Assoc J. 2003;169(9):921-4.

9. Statistics Korea, Causes of death. http://kosis.kr/statHtml/statHtml.do?orgld= 101\&tblld=DT_1B34E01\&conn_path=I2 (2004-2014). Accessed 10 June 2016.

10. Park H-K. Nutrition policy in South Korea. Asia Pac J Clin Nutr. 2008:17(S1):343-5.

11. Cowburn G, Stockley L. Consumer understanding and use of nutrition labelling: a systematic review. Public Health Nutr. 2005;8(01):21-8.

12. Guthrie JF, Fox JJ, Cleveland LE, Welsh S. Who uses nutrition labeling, and what effects does label use have on diet quality? J Nutr Educ. 1995;27(4):163-72.

13. Satia JA, Galanko JA, Neuhouser ML. Food nutrition label use is associated with demographic, behavioral, and psychosocial factors and dietary intake among African Americans in North Carolina. J Am Diet Assoc. 2005;105(3): 392-402.

14. Campos S, Doxey J, Hammond D. Nutrition labels on pre-packaged foods: a systematic review. Public Health Nutr. 2011:14(08):1496-506.

15. Musunuru K. Atherogenic dyslipidemia: cardiovascular risk and dietary intervention. Lipids. 2010;45(10):907-14.

16. McPherson R, Frohlich J, Fodor G, Genest J. Canadian Cardiovascular Society position statement-recommendations for the diagnosis and treatment of dyslipidemia and prevention of cardiovascular disease. Can J Cardiol. 2006; 22(11):913-27.

17. Friedewald WT, Levy RI, Fredrickson DS. Estimation of the concentration of low-density lipoprotein cholesterol in plasma, without use of the preparative ultracentrifuge. Clin Chem. 1972;18(6):499-502.

18. Wadwa RP, Kinney GL, Maahs DM, Snell-Bergeon J, Hokanson JE, Garg SK, Eckel RH, Rewers M. Awareness and treatment of dyslipidemia in young adults with type 1 diabetes. Diabetes Care. 2005;28(5):1051-6.

19. Assmann G, Schulte H. The Prospective Cardiovascular Münster (PROCAM) study: prevalence of hyperlipidemia in persons with hypertension and/or diabetes mellitus and the relationship to coronary heart disease. Am Heart J. 1988;116(6):1713-24.

20. Graffagnino C, Gasecki AP, Doig GS, Hachinski VC. The importance of family history in cerebrovascular disease. Stroke. 1994;25:1599.

21. Yancy WS, Olsen MK, Guyton JR, Bakst RP, Westman EC. A low-carbohydrate, ketogenic diet versus a low-fat diet to treat obesity and hyperlipidemia: a randomized, controlled trial. Ann Intern Med. 2004;140(10):769-77.

22. Stefanick ML, Mackey S, Sheehan M, Ellsworth N, Haskell WL, Wood PD. Effects of diet and exercise in men and postmenopausal women with low levels of HDL cholesterol and high levels of LDL cholesterol. N Engl J Med. 1998;339(1):12-20

23. Chung SJ, Kim JH, Lee JS, Lee DH, Kim SH, Yu CH. A suggestion to develop a nutrition policy on food and nutrition labeling and education systems for fast food and carbonated soft drinks in Korea. Korean J Nutr. 2004; 37(5):394-405

24. Von Lengerke $T$, Vinck J, Rütten A, Reitmeir P, Abel T, Kannas L, LüSchen $G$, Diaz JAR, Van Der Zee J. Health policy perception and health behaviours: A multilevel analysis and implications for public health psychology. J Health Psychol. 2004;9(1):157-75.

25. Levy AS, Fein SB. Consumers' ability to perform tasks using nutrition labels. J Nutr Educ. 1998;30(4):210-7.

26. Shine A, O'Reilly S, O'Sullivan K. Consumer attitudes to nutrition labelling. Br Food J. 1997;99(8):283-9.

27. Mielck A: Perception of health inequalities in different social classes, by health professionals and health policy makers in Germany and the United Kingdom. In.: WZB Discussion Paper; 1998.

28. Scharnagl H, Nauck M, Wieland H, März W. The Friedewald formula underestimates LDL cholesterol at low concentrations. Clin Chem Lab Med. 2001;39(5):426-31.

\section{Submit your next manuscript to BioMed Central and we will help you at every step:}

- We accept pre-submission inquiries

- Our selector tool helps you to find the most relevant journal

- We provide round the clock customer support

- Convenient online submission

- Thorough peer review

- Inclusion in PubMed and all major indexing services

- Maximum visibility for your research

Submit your manuscript at www.biomedcentral.com/submit

) Biomed Central 